\title{
Monitoramento da carga interna competitiva de uma equipe de basquetebol sub-17 durante os jogos escolares regionais
}

\author{
Training load and signs and symptoms stress in \\ school sub17 during basketball school games
}

Julio Cesar Barbosa de Lima Pinto, ${ }^{1}$ Tancredo Cesar Barbosa Menezes, ${ }^{1}$ Renêe de Caldas Honorato, Arnaldo Luis Mortatti. ${ }^{1}$

'Universidade Federal do Rio Grande de Norte (UFRN), Natal, RN, Brasil.

Recebido em: janeiro 2015 / Aceito em: fevereiro 2015

julioduibmx@gmail.com

\section{RESUMO}

Objetivo: monitorar a carga interna competitiva por meio da percepção subjetiva de esforço da sessão (PSE-sessão) e avaliar a tolerância ao estresse em escolares sub-17, durante três jogos consecutivos de basquetebol. Método: a amostra foi composta por dez jovens jogadores de basquetebol $(16,2 \pm 0,78$ anos; $173,8 \pm 6,77 \mathrm{~cm} ; 68,3 \pm 9,80 \mathrm{~kg}$ ). A variação dos sinais e sintomas de estresse foi verificada por meio do questionário Daily Analysis of Life Demands in Athletes (DALDA). Foram quantificadas a duração das partidas e a PSE-sessão para determinação da carga interna. Resultados: foi verificada diferença significativa na carga interna competitiva dada pela PSE da sessão, do jogo um para o jogo três $(p<0,001)$ e do jogo 2 para o jogo $3(p=0,045)(F=9,94)$; já o número de respostas "piores que o normal" dos sinais $(p=0,590)$ e sintomas de estresse ( $p=0,910)$ não diferiram entre os momentos pré e pós-competição. Considerações finais: foi observado que a carga interna competitiva obtida pela PSE da sessão teve os valores aumentados proporcionalmente em função do acúmulo dos jogos realizados, demonstrando que esse instrumento pode ser sensível para determinar a carga interna competitiva em jovens atletas escolares sub-17.

Palavras-chave: Competição Escolar; Controle de Treinamento; PSE da sessão; DALDA.

\section{ABSTRACT}

Objective: monitor the competitive internal load through session-rating of perceived exertion (session-RPE) method, and the signs and symptoms of stress in school children under-17 during three consecutive basketball matches. Method: sample consisted of ten basketball players (16.2 \pm 0.78 years; $173.8 \pm 6.77$ $\mathrm{cm} ; 68.3 \pm 9.80 \mathrm{~kg}$ ). Stress signs and symptoms variation were verified by the Daily Analysis of Life Demands in Athletes questionnaire (DALDA). The length of matches and session-RPE were quantified for monitoring the internal load. Results: training load to the values of three arbitrary units differ from one game play significantly ( $p<0.001)$, two ( $p<0.045)$; since the number of answers "worse than usual" signs ( $p=0.590)$ and stress symptoms $(p=0.910)$ did not differ from time pre and post-competition. Final considerations: was observed that competitive internal load obtained by the session-RPE had progressive income because of consecutive games. Session-RPE seems to be a viable tool in control and understanding of school youths training load in sub 17 category. Already the signs and symptoms stress did not change the effect of the games.

Keywords: School Competition; Training Control; Session-RPE; DALDA. 


\section{INTRODUÇÃO}

O crescimento e a valorização do esporte mundial nas últimas décadas proporcionaram um favorecimento ao surgimento de equipes de treinamento com crianças nas mais diversas modalidades de esportes ${ }^{1}$. Durante o processo de formação esportiva, a performance do jovem em eventos competitivos é considerado como o principal fator relacionado ao sucesso. Esse desempenho é caracterizado pela junção de fatores multivariados e interdependentes dos quais podemos destacar as capacidades física, técnica, tática e psicológica ${ }^{1}$.

A teoria do treinamento ressalta a importância de desenvolver fortes competências básicas com a finalidade de aumentar a capacidade de trabalho, a eficácia de habilidades e as qualidades psicológicas necessárias para melhorar o desempenho do atleta e alcançar o objetivo específico em competições ${ }^{2}$. Um plano de treinamento eficaz no esporte tem o papel de melhorar o desempenho esportivo, para isso tem que envolver um equilíbrio constante entre o estresse e a recuperação. Em contraste, a acumulação de tensão/fadiga acompanhada por regeneração insuficiente é uma das principais causas de excesso de treino ${ }^{3,4}$.

Assim, o monitoramento da carga de treino pode ser importante para identificar as adaptações induzida pelo estresse fisiológico, acarretado pelo treinamento físico em função da manipulação do volume e intensidade de treinamento, demonstrado em termos do seu resultado por meio de adaptações anatômicas, fisiológicas, bioquímicas, funcionais ${ }^{5}$. Embora se tenha algumas técnicas para monitoramento dos atletas em rotinas de treino ou em momentos competitivos, pouco se sabe a respeito da carga de treinamento em adolescentes escolares durante competições esportivas.

O método PSE da sessão proposto por Foster et al. ${ }^{6,7}$ vem sendo utilizado para a quantificação da carga de treinamento mostrando-se eficiente em esportes coletivos $^{8,9}$ e individuais ${ }^{10}$, assim como esportes de combate ${ }^{11}$. Outros trabalhos de Borresen, \& Lambert ${ }^{12}$ e Haddad et al. ${ }^{11}$ demonstraram a validação do método de PSE da sessão comparados com meios objetivos que se baseiam no somatório de zonas de frequência cardíaca (TRIMP).

Além disso, alguns estudos propõem a utilização de questionários para a detecção da tolerância ao estresse, no intuito de monitorar sinais e sintomas de estresse decorrentes das rotinas de treinamento ou durante a competição ${ }^{13,14}$, pois, quando o atleta é submetido a rotinas de treinamento intenso ou competições no intuito de obter resultados máximos do organismo ou na competição, o corpo do atleta passa por ciclos de fadiga e recuperação tendo interferência em suas capacidades físicas, gerando alterações em sintomas de estresse ${ }^{14}$.

Nesse sentido, muitas pesquisas ${ }^{15-18}$ estão utilizando o questionário Daily Analysis of Life Demands in Athletes $(\text { DALDA })^{19}$ na tentativa de detectar os atletas em overreached, assim como, utilizando-o como um sistema de alerta para o possível aparecimento de overtraining.

Na literatura atual, a utilização de questionário em conjunto com métodos indiretos para medir carga de treinamento vem ganhando respaldo significativo para o acompanhamento do treinamento ${ }^{20-22}$. A maioria das investigações inerentes ao comportamento, carga de treinamento e do estresse piscicofisiológico são é reali- zadas em indivíduos adultos. Nesse sentido, o objetivo do trabalho foi analisar a carga de treinamento por meio da PSE da sessão e os sinais e sintomas de estresse em jovens escolares, durante três dias de jogos consecutivos da fase final dos Jogos Escolares do Estado do Ceará, na modalidade basquetebol. A hipótese inicial da pesquisa foi que a realização dos jogos consecutivos poderia acarretar em um aumento da carga interna e que esse aumento poderia influenciar na tolerância ao estresse dos jovens jogadores.

\section{MÉTODO}

\section{Amostra}

Participaram do estudo dez jovens escolares jogadores de basquetebol $(16,2 \pm 0,78$ anos; 173,8 \pm $6,77 \mathrm{~cm}$ de altura; 68,3 $\pm 9,80 \mathrm{~kg}$ de massa corporal), que disputaram a fase estadual dos Jogos Escolares do Ceará categoria 15 a 17 anos, no período de 17 a 19 de outubro de 2014. Todos tinham uma frequência semanal de três a quatro sessões de treinamento, durante a semana com duração de 90 minutos, envolvendo componentes técnico e tático específico.

\section{Delineamento experimental}

O estudo foi realizado na cidade de Fortaleza, no estado do Ceará - Brasil, com adolescentes de uma escola particular que participavam da fase final dos Jogos Escolares do Ceará organizados pela Secretaria do Esporte do Estado do Ceará. Solicitou, ao responsável pela equipe escolar, a autorização para a realização dos questionários e explicitado aos adolescentes o caráter voluntário da participação, bem como o objetivo do trabalho, a maneira de preenchimento e o anonimato das informações adquiridas. O horário e o local dos jogos foram definidos pela Federação Universitária Cearense de Esportes (FUCE), sendo o primeiro jogo (P1) realizado às 15:00 horas do dia 17/10/2014; o segundo (P2) as 14:00 horas do dia 18/10/2014 e o último jogo (P3) as 8:00 horas do dia 19/10/2014.

\section{Procedimento}

A coleta dos dados foi realizada por etapas (figura 1): a) foram coletados os dados peso, altura e idade para caracterização do grupo; b) aplicou-se o questionário DALDA nos momentos pré e pós-competição e c) foram verificados o tempo de jogo e a PSE da sessão de cada partida de acordo com as normas de Foster et al. ${ }^{7}$. Todos os jogos foram precedidos de 5 minutos de aquecimento. 0 tempo de cada partida foi previamente definida em 40 minutos, divididos em 4 tempos de 10 minutos com intervalo de 1 minutos, entre o primeiro e segundo, terceiro e quarto tempo. Já, o tempo entre o segundo e terceiro foram 3 minutos de intervalo.

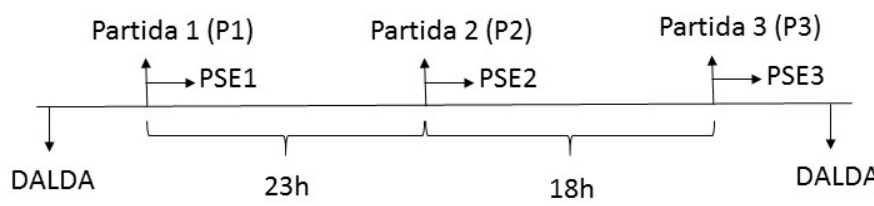

Figura 1 - estrutura temporal da coleta de dados durante a competição. 


\section{DALDA}

O questionário para análise dos sintomas de estresse foi preenchido, primeiramente, antes de iniciar os jogos e ao final dos jogos, conforme procedimentos utilizados por Robson-Ansley, Blannin e Gleeson ${ }^{18}$. O DALDA é dividido em duas partes - parte A que representa as fontes de estresse e parte B que representa sintomas de estresse. Este instrumento requer que o atleta assinale cada variável, em cada parte do questionário ( $A$ e $B$ ), como sendo "pior do que o normal", "normal", ou "melhor do que o normal" em função da sua percepção das fontes e sintomas de estresse. Os indivíduos já possuíam experiência prévia com a utilização do instrumento.

\section{Determinação da Carga Interna}

A determinação da carga interna competitiva foi realizada a partir da coleta da percepção subjetiva do esforço da sessão; foram seguidos os procedimentos de Foster et al. ${ }^{7}$. Para a quantificação da carga interna competitiva $(\mathrm{CIC})$ foi realizado o cálculo do produto da duração do jogo realizado pelo valor obtido pela escala da percepção subjetiva do esforço. Os valores encontrados foram expressos em unidades arbitrárias (UA) ${ }^{23}$.

\section{Análise estatística}

Estatística descritiva com média e desvio padrão foram aplicados nos resultados para caracterização da amostra e os valores PSE da sessão, unidades arbitrárias e respostas "piores que o normal" do questionário DALDA. A normalidade das três variáveis foi verificada pelo teste de Shapiro-Wilk e Kolmogorov-Smirnov. Devido a não violação da normalidade dos dados, ANOVA de medidas repetidas com post hoc de Bonferroni foi utilizada para comparação dos valores percepção subjetiva de esforço e valores carga interna por meio da PSE da sessão nos três jogos. Na comparação dos valores do questionário DALDA foi utilizado Test $-\mathrm{t}$ para comparar os momentos pré e pós. O programa para análise estatística GraphPad Prism 5 e o SPSS 20.0 forma utilizados. $O$ nível de significância foi $p<0,05$.

\section{RESULTADOS}

Os valores da PSE da sessão e da carga interna competitiva são apresentados na tabela 1 e a figura 2 mostra a carga interna competitiva dada pela PSE da sessão. Houve diferenças significativas das duas primeira partidas em relação a partida três, nas duas variáveis.

Tabela 1 - Variação da carga interna de treinamento ao longo dos jogos

\begin{tabular}{cccc}
\hline & P1 & P2 & P3 \\
\hline PSE (UA) & $3,55(1,6)$ & $5,15(2,1)$ & $7,60(2,9)^{* \#}$ \\
Carga Interna (UA) & $177,5(80,3)$ & $257,5(106,8)$ & $380(145,7)^{* \#}$ \\
\hline Unidades arbitrárias (UA); Média (desvio padrão); * $=$ diferença significativa em \\
relação a P1 ( $p=0,00) ;$ \# = diferença significativa em relação a $P 2(p<0,045)$.
\end{tabular}

Com relação aos valores do questionário DALDA, o número de respostas "piores que o normal", tanto da parte $A$, como da parte $B$ do questionário não diferiram significativamente comparando o momento pré e pós (tabela 2).

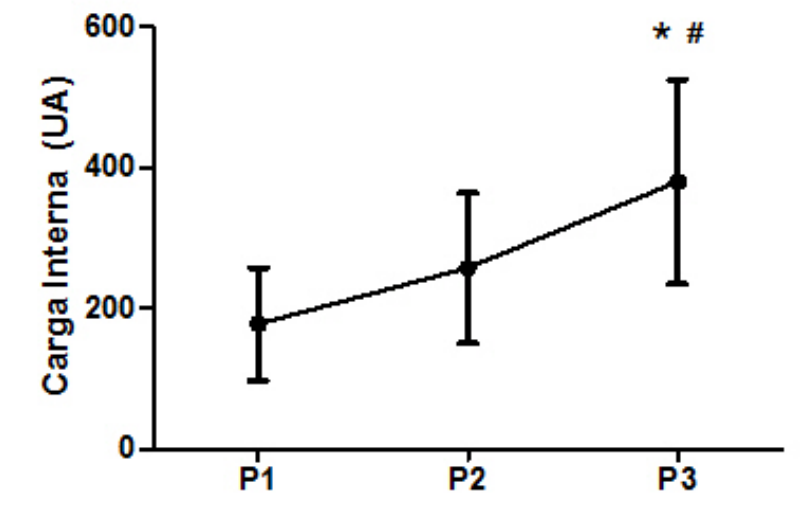

Unidades arbitrárias $(U A) ;{ }^{*}=$ diferença significativa em relação a $P 1(p=0,00)$; \# = diferença significativa em relação a P2 $(p<0,045)$.

Figura 2 - Carga Interna Competitiva.

Tabela 2 - Comparação dos valores das respostas "pior que o normal" nas partes $A$ e $B$ do questionário DALDA no momento pré e pós jogos.

\begin{tabular}{cccc}
\hline & Pré & Pós & P valor \\
\hline Parte A & $1,4(1,34)$ & $1,3(1,56)$ & 0,59 \\
Parte B & $4,1(3,44)$ & $4,0(4,21)$ & 0,91 \\
\hline
\end{tabular}

\section{DISCUSSÃO E CONSIDERACִÕES FINAIS}

O objetivo deste estudo foi analisar a dinâmica dos sinais e sintomas de estresse e da carga interna competitiva em uma equipe de basquetebol sub-17. O principal achado do trabalho foi a identificação do aumento da carga interna competitiva em função do acúmulo dos jogos e em relação aos valores dos sinais e sintomas de estresse; esses não se alteraram significativamente após a realização dos jogos. Portanto, nossa hipótese inicial confirmou-se parcialmente, uma vez que, era esperado que os sinais e sintomas de estresse fossem modulados pela realização dos três jogos consecutivos.

Os valores carga interna competitiva, verificada pela PSE da sessão, durante os três jogos consecutivos, mostraram congruência com o estudo de Nunes et al. $^{24}$ que encontraram valores médios de $321( \pm 127)$ UA. Esses valores estão abaixo daqueles considerados intensos (intensificação deliberada das cargas de treino) para a população adulta (acima de 400 UA) $)^{5,17,25}$.

Apesar de não alcançarem valores intensos de carga competitiva, o aumento progressivo da carga interna nos jogos consecutivos em jovens atletas pode demonstrar um efeito do processo recuperativo insuficiente. Sugerindo que com a progressão dos jogos, juntamente com adversários mais difíceis, associado com um maior estresse psicológico e algum nível de fadiga acumulada, os valores de percepção subjetiva de esforço aumentaram de uma forma linear, como nos achados de Mortatti, et al. ${ }^{26}$.

Esse aumento pode ser explicado pelo curto intervalo entre as partidas (ver figura1), principalmente entre $\mathrm{P} 2$ e P3, que foi apenas 17 horas. O pouco tempo de descanso pode ter levado a uma baixa recuperação dos sistemas energéticos, uma vez que a alta demanda fisiológica, com evidências mostrando que jogadores 
sub-19, durante a partida, apresentam média de frequência cardíaca de $91 \%$ da frequência cardíaca máxima, também, pela pressão psicológica que a disputa de um campeonato pode $\operatorname{trazer}^{27,28}$, além de um possível aumento da dor muscular, que são fatores que podem desencadear sensações relevantes, para os atletas reportarem valores maiores para a PSE da sessão ${ }^{13}$.

Além disso, a P3 (derrota) foi contra um adversário de nível conhecidamente superior aos da P2 (vitória) e P1 (vitória), o que pode ter tornado o jogo mais cansativo em comparação aos outros que foram vencidos com uma grande vantagem no placar $^{28}$.

Essa condição de competição, com intervalo reduzido entre os jogos, pode ser um fator importante de análise para comissão técnica, pois, mesmo observando que, a magnitude das cargas não foram tão intensas, quando comparadas com de adultos $^{5}$ e com a de adolescentes basquetebolistas, que foram submetidas a semana de intensificação de treino ${ }^{25}$, o aumento progressivo dos valores da PSE da sessão, visto durante os jogos sucessivos, pode provocar um desgaste fisiológico dos sistemas energéticos e estruturas osteomusculares, que facilitam a ocorrência de lesões e o desencadeamento do processo de overtraining ${ }^{3}$. Por isso, é importante usar ferramentas para controle da carga interna, tanto de treinamento, como competitiva, pois em muitas ocasiões os períodos preparatório e competitivo são próximos ou até mesmo intercalados ao longo do ano e podem gerar grande repercussão na saúde e desempenho do atleta se não forem bem controlados.

No grupo estudado, os sinais e sintomas de estresse não tiveram valores significativamente diferentes do momento pré para o pós-competitivo; diferente dos resultados encontrados por Gomes et al. ${ }^{29}$ e Moreira et al. ${ }^{25}$ que observaram modificações dos sinais e sintomas de estresse em jovens atletas de diferentes modalidades, porém analisados semanalmente. A manutenção dos níveis dos sinais e sintomas de estresse não é compatível com situações de competições esportivas, pois essas provocam estressores psicológicos adicionais associados com exigências físicas, maximizando as respostas hormonais de estresse, como cortisol. Além disso, a competição pode ser caracterizada como uma situação que envolve ego, novidade, ansiedade, falta de controle e imprevisibilidade, podendo levar a propriedades afetivas negativas ${ }^{30}$. Porém, como o estresse é composto por variáveis muito subjetivas, os resultados podem variar de acordo com a experiência anterior dos sujeitos com eventos competitivos, o tipo de trabalho psicológico que os atletas recebem para conseguir criar estratégias de controle do estresse (coping), qual o nível de cobrança que os jogadores tem para aquela competição analisada, o nível de motivação e outras razões que podem modificar as respostas emocionais associadas ao estresse ${ }^{31-33}$.

Em conclusão, a carga interna competitiva teve efeito progressivo em função dos três jogos consecutivos, da mesma forma que a percepção subjetiva de esforço, demonstrando que este tipo de competição pode promover uma demanda fisiológica e psicológica que pode refletir no desempenho de jovens escolares que estão inseridos em programas de treinamento sistemático de alguma modalidade esportiva. No entanto, para os sinais e sintomas de estresse não houve modificação do período pré para o período pós-competitivo, sendo necessários mais estudos em uma população semelhante para obter maiores esclarecimentos. O monitoramento da carga interna competitiva em jogos oficiais pode contribuir com informações para a comissão técnica no planejamento/periodização das cargas de treinamento em momentos próximos de competições. Porém, apesar da importância notável dessas ferramentas de controle de carga, a perspicácia do treinador tem ainda uma função primordial na decisão de quais atletas estão aptos para jogar, principalmente com atletas em formação nas categorias de base, que costumam não ter uma experiência grande em competições e muitas vezes, motivados pela disputa, escondem o cansaço e querem permanecer na partida o maior tempo possível para ajudar o time. É necessário que haja um bom planejamento com a utilização de estratégia durante a competição para que alguns atletas não sejam sobrecarregados nos primeiros jogos e desfalquem a equipe nos jogos finais, que geralmente são decisivos neste tipo de competição.

\section{CONFLITO DE INTERESSES}

Todos os autores declararam não haver qualquer potencial conflito de interesses referente a este artigo.

\section{REFERÊNCIAS}

1. Ré AHN, De Rose Jr. D, Böhme MTS. Stress and Competitive Level: Considerations about Youth Futsal Players. Revista Brasileira Ciência e Movimento, 2004; 12(4): 83-87.

2. Brunelli DT, Borin JP, Rodrigues A, Bonganha V, Prestes J, Montagner PC, et al. Immune responses, upper respiratory illness symptoms, and load changes in young athletes during the preparatory period of the training periodization. Open access journal of sports medicine, 2012; 3: 43-49.

3. Coutts AJ, Slattery KM, Wallace LK. Practical tests for monitoring performance, fatigue and recovery in triathletes. J Sci Med Sport, 2007; 10(6): 372-381.

4. di Fronso S, Nakamura FY, Bortoli L, Robazza C, Bertollo M. Stress and Recovery Balance in Amateur Basketball Players: Differences by Gender and Preparation Phase. Int J Sport Physiol, 2013; 8(6): 618-22.

5. Impellizzeri FM, Rampinini E, Coutts AJ, Sassi A, Marcora SM. Use of RPE-based training load in soccer. Med Sci Sport Exer, 2004; 36(6): 1042-1047.

6. Foster C, Florhaug JA, Franklin J, Gottschall L, Hrovatin LA, Parker S, et al. A new approach to monitoring exercise training. J Strength Cond Res, 2001; 15(1): 109-115.

7. Foster C, Hector LL, Welsh R, Schrager M, Green MA, Snyder AC. Effects of Specific Versus Cross-Training on Running Performance. Eur J Appl Physiol O, 1995; 70(4): 367-372.

8. Alexiou $\mathrm{H}$, Coutts AJ. A Comparison of Methods Used for Quantifying Internal Training Load in Women Soccer Players. Int J Sport Physiol, 2008; 3(3): 320-330.

9. Coutts AJ, Reaburn PRJ, Murphy AJ, Pine MJ, Impellizzeri FM. Validity of the session-RPE method for determining training load in team sport athletes. J Sci Med Sport, 2003; 6(4): 525.

10. Wallace L, Coutts A, Bell J, Simpson N, Slattery K. Using Session-RPE to Monitor Training Load in Swimmers. Strength 
Cond J, 2008; 30(6): 72-76.

11. Haddad M, Chaouachi A, Castagna C, Wong DP, Behm DG, Chamari K. The Construct Validity of Session RPE During an Intensive Camp in Young Male Taekwondo Athletes. Int J Sport Physiol, 2011; 6(2): 252-263.

12. Borresen J, Lambert MI. Quantifying Training Load: A Comparison of Subjective and Objective Methods. Int $\mathrm{J}$ Sport Physiol, 2008; 3(1): 16-30.

13. Rushall BS. A tool for measuring stress tolerance in elite athletes. Journal of Applied Sport Psychology, 1990; 2(1): 51-66.

14. Moreira A, Freitas CG, Nakamura FY, Aoki MS. Session RPE and stress tolerance in young volleyball and basketball players. Brazilian Journal of Kinanthropometry and Human Performance, 2010; 12(5): 345-351.

15. Moreira A, Cavazzoni PB. Monitorando o treinamento atrvés do Wisconsin Upper Respiratory Sympton Survey-21 e Dailly Analysis of Live Demands in athletes. Revista da Educação Física/UEM, 2009; 20(1): 109-119.

16. Mortatti AL, Coelho AAC, Costa EC. Monitoramento da carga interna de treinamento, tolerância ao estresse e ocorrência de infecções em jovens atletas de futebol. Revista da Educação Física/UEM, 2014, 25(4): 629-638.

17. Coutts AJ, Slattery KM, Wallace LK. Practical tests for monitoring performance, fatigue and recovery in triathletes. J Sci Med Sport, 2007; 10(6): 372-381.

18. Freitas CG, Aoki MS, Franciscon CA, Arruda AF, Carling C, Moreira A. Psychophysiological responses to overloading and tapering phases in elite young soccer players. Pediatr Exerc Sci, 2014; 26(2): 195-202.

19. Robson-Ansley PJ, Blannin A, Gleeson M. Elevated plasma interleukin- 6 levels in trained male triathletes following an acute period of intense interval training. Eur J Appl Physiol, 2007; 99(4): 353-360.

20. Halson SL. Monitoring Training Load to Understand Fatigue in Athletes. Sports Med, 2014; 44(2): 139-147.

21. Manzi V, D'Ottavio S, Impellizzeri FM, Chaouachi A, Chamari K, Castagna C. Profile of Weekly Training Load in Elite Male Professional Basketball Players. J Strength Cond Res, 2010; 24(5): 1399-406.

22. Moreira A, Arsati F, Lima-Arsati YBD, Simoes AC, de Araujo VC. Monitoring Stress Tolerance and Occurrences of Upper Respiratory Illness in Basketball Players by Means of Psychometric Tools and Salivary Biomarkers. Stress Health,
2011; 27(3): 166-172.

23. Foster $\mathrm{C}$. Monitoring training in athletes with reference to overtraining syndrome. Med Sci Sports Exerc, 1998; 30(7): 1164-1168.

24. Nunes JA, Costa EC, Viveiros L, Moreira A, Aoki MS. Monitoring internal load in basketball. Brazilian Journal of Kinanthropometry and Human Performance, 2011; 13(1): 67-72.

25. Moreira A, Nakamura F, Cavazzoni P, Gomes J, Martignago P. O efeito da intensificação do treinamento na percepção de esforço da sessão e nas fontes e sintomas de estresse em jogadores jovens de basquetebol. Revista da Educação Física/UEM, 2010; 21(2); 287-296.

26. Mortatti AL, Moreira A, Aoki MS, Crewther BT, Castagna C, de Arruda AF, et al. Effect of competition on salivary cortisol, immunoglobulin $A$, and upper respiratory tract infections in elite young soccer players. J Strength Cond Res, 2012; 26(5): 1396-1401.

27. Ben Abdelkrim N, El Fazaa S, El Ati J. Time-motion analysis and physiological data of elite under19-year-old basketball players during competition. Brit J Sport Med, 2007; 41(2): 69-75.

28. Moreira A, McGuigan MR, Arruda AFS, Freitas CG, Aoki MS. Monitoring Internal Load Parameters during Simulated and Official Basketball Matches. J Strength Cond Res, 2012; 26(3): 861-866

29. Gomes RV, Moreira A, Lodo L, Nosaka K, Coutts AJ, Aoki MS. Monitoring Training Loads, Stress, Immune-Endocrine Responses and Performance in Tennis Players. Biol Sport, 2013; 30(3): 173-180.

30. Moreira A, Freitas CG, Nakamura FY, Drago G, Drago M, Aoki MS. Effect of Match Importance on Salivary Cortisol and Immunoglobulin a Responses in Elite Young Volleyball Players. J Strength Cond Res, 2013; 27(1): 202-7.

31. Calmeiro L, Tenenbaum G, Eccles DW. Managing pressure: patterns of appraisals and coping strategies of non-elite and elite athletes during competition. J Sport Sci, 2014; 32(19): 1813-1820.

32. Martinent G, Ferrand C. A Field Study of Discrete Emotions: Athletes' Cognitive Appraisals During Competition. Research quarterly for exercise and sport, 2014; 86(1), 51-62.

33. Nicholls AR, Perry JL, Calmeiro L. Precompetitive achievement goals, stress appraisals, emotions, and coping among athletes. Journal of sport \& exercise psychology, 2014; 36: 433-445. 